\title{
Right atrial appendage: an important structure to drive atrial fibrillation
}

\author{
Yang Liu ${ }^{1} \cdot$ Ziliang Song $^{1} \cdot$ Weifeng Jiang ${ }^{1} \cdot$ Shaohui $\mathrm{Wu}^{1} \cdot \mathrm{Xu} \mathrm{Liu}^{1}{ }^{1} \cdot \mathrm{Mu} \mathrm{Qin}^{1}$
}

Received: 12 October 2021 / Accepted: 27 December 2021 / Published online: 18 February 2022

(C) The Author(s) 2022

\begin{abstract}
Purpose Understanding of the atrial fibrillation (AF) driven by right atrial appendage (RAA) is limited. This study aimed to understand the characteristics of the AF driven by RAA and explore ablation methods.

Methods This was a retrospective study and patients who were identified as having the AF driven by RAA were reviewed. Ablation was performed during AF. Potential maps of the left and right atrium, electrophysiological examinations, and ablation methods were studied.

Results Among the 20 identified patients (mean age 67.0 \pm 11.2 years; ejection fraction $62.9 \pm 6.0 \%$; LA diameter $43.1 \pm 4.9 \mathrm{~mm}$; RA diameter $51.7 \pm 8.3 \times 42.9 \pm 3.7 \mathrm{~mm})$, the AF cycle length in RAA $(134.0 \pm 10.9 \mathrm{~ms})$ was the shortest, and the fastest frequency potentials were located in the RAA in $65 \%$ of patients. For the left atrium, the AF cycle length of the roof $(145.5 \pm 14.9 \mathrm{~ms})$ was the shortest, followed by the left atrial appendage $(153.7 \pm 17.1 \mathrm{~ms})$ and bottom $(154.8 \pm 11.8 \mathrm{~ms})$. High-frequency potentials of RAA could be rapidly conducted to left atrium via sagittal bundle and Bachmann's bundle, and the conduction time $(55.0 \pm 5.0 \mathrm{~ms})$ was significantly shorter than the mean bi-atrial activation time $(176.7 \pm 10.3 \mathrm{~ms}$, $P<0.0001)$. AF could be terminated after ablation at the RAA base (17 patients) or mechanical stimulation within the RAA (3 patients). To date, only two patients had recurrent atrial flutter, while the remaining patients maintained sinus rhythm. Conclusion The AF driven by RAA is characterized by high-frequency potentials in RAA, and ablation at the RAA base can achieve a satisfactory therapeutic effect.
\end{abstract}

Keywords Atrial fibrillation $\cdot$ Right atrial appendage $\cdot$ Potential maps $\cdot$ Catheter ablation

\section{Introduction}

Yang Liu and Ziliang Song contributed equally to this work

Xu Liu

heartlx@sina.com

$\triangle \mathrm{Mu}$ Qin

qinmuae@163.com

Yang Liu

dococean@163.com

Ziliang Song

drsongzl@126.com

Weifeng Jiang

jiangweifeng83@sina.com

Shaohui Wu

wushaohui18@163.com

1 Department of Cardiology, Shanghai Chest Hospital, Shanghai Jiao Tong University, 241 Huaihai West Road, Shanghai, China
In a previous study, the incidence of right atrial appendage (RAA) as a driver of atrial fibrillation (AF) was very low [1]. Subsequently, the special anatomy of RAA was focused by several studies and it was speculated to be related to the mechanism of arrhythmia. Recently, Ghannam et al. found that the RAA appeared to be a major source of fibrillation activity in the right atrium (RA) [2]. In addition, several basic studies also demonstrated that tissue heterogeneity of the pectinate structure in the RAA itself provided the conditions for the formation and maintenance of $\mathrm{AF}$ driver [3, 4]. However, there is still a lack of clinical evidences on the AF driven by RAA. We found that the proportion of AF mediated by RAA was not uncommon if routine bi-atrial mapping was performed during the procedure. The present study summarizes the characteristics of potentials and the ablation methods in the AF driven by RAA, and hopes to be able to help in the identification and treatment of this type of AF. 


\section{Methods}

\subsection{Study population}

This was a retrospective and observational study at Shanghai Chest Hospital between March 2019 and April 2021, and patients with the AF driven by RAA were examined. Patients were included if their AF could be terminated (sinus rhythm or atrial flutter/atrial tachycardia) through intervening RAA during the procedure. Informed consent was not necessary due to the observational nature of the study. This study protocol was approved by the Institutional Ethics Committee at the Shanghai Chest Hospital.

\subsection{Mapping and catheter ablation}

All patients were prepared preoperatively depending on the guidelines. A decapolar catheter was placed into the coronary sinus (CS), and the activated clotting time (ACT) was maintained between 300 and 350 s during the procedure. After two punctures of atrial septum, left atrium model was constructed with the use of a PentaRay catheter and the Carto 3 system (Biosense-Webster). All patients underwent circumferential pulmonary vein isolation (CPVI) first during AF. The SMARTTOUCH Catheter or the THERMOCOOL SMARTTOUCH SF Catheter (Biosense Webster, Inc., Irvine, California) were used for ablation. Bi-atrial mapping was performed using a PentaRay catheter if AF was not terminated after CPVI. According to the previous literature, reference AF cycle length (AFCL) calculated manually by averaging 10 consecutive beats [5] was used to analyze the potentials in different sites of atria. The main part of the RAA was constructed using a PentaRay catheter in FAM (fast anatomical mapping) mode, and intracardiac echocardiography (ICE) was also utilized to further determine the ostium of the RAA. AF was identified to be possibly driven by RAA if highfrequency potentials were mapped inside or at the base of RAA. The high-frequency potentials were considered to be potentials with AFCLs equal to or shorter than left atrial potentials AFCLs. For the patients with similar frequencies of RAA and left atrial potentials, we initially ablated the left atrium in regions with relatively shorter AFCLs. If these procedures did not affect the condition of AF (termination or slowing of the AFCL), we performed the ablation of RAA. If the RAA potential had shorter AFCL compared with left atrial potentials, ablation of the RAA was performed, and no additional ablation of the left atrium was performed except for CPVI. For cases where the fastest frequency potentials were at the RAA base, ablation was performed in sheet form at the corresponding location of the base. For cases where the fastest frequency potentials were inside the RAA, linear ablation was performed on the lateral and septal sides of the base. The septal side was ablated to the superior vena cava (SVC) ostium, and the lateral side was ablated from the middle of the RAA vestibule to tricuspid valve (TV) after ICE identified the RAA ostium. If AF was terminated to atrial flutter (AFL)/ atrial tachycardia (AT), the latter was mapped and ablated. Patients underwent transthoracic cardioversion if their AFL/AT could not be terminated after ablation.

\subsection{Electrophysiological study}

To elucidate the pro-arrhythmic substrate of RAA, we used ICE and pacing mapping to study the anatomical structure and electrical conductivity characteristics in RAA under sinus rhythm. Multiple ultrasonic sectors of the RAA were obtained by moving the ultrasonic catheter in the right atrium. The contour of the RAA and the important structures including the sagittal bundle (SB) and terminal crest (TC) were constructed and shown in different colors under different ultrasound sectors. Then, the ablation catheter was placed at a specific location in the RAA and the pacing was initiated, while the local activation time (LAT) mapping was utilized to show the sequence of activation conduction in the right atrium. Finally, we inserted the PentaRay catheter into the left atrium and used the same method described above to explore the sequence of activation conduction from the RAA to the left atrium. The pacing current output was $10 \mathrm{~mA}$.

\subsection{Follow-up}

After ablation, the patients were hospitalized for at least 3 days and received continuous ECG monitoring during the first 48 h. At 1, 3, 6, 9, and 12 months after ablation, the patients went to the outpatient clinic for reexamination and received 24-h Holter monitoring. If follow-up information for a patient was not available in the hospital's medical record system, we contacted the patient by phone. Patients were not routinely treated with antiarrhythmic drugs after ablation unless recurrence occurred. Recurrence was defined as sustained AF/AFL/AT after the 3-month blanking period.

\subsection{Statistical analysis}

The data were presented as mean \pm SD and median \pm IQ for continuous variables, as appropriate. Continuous variables among different groups were compared by a one-way ANOVA test, and a $P$ value $<0.05$ was considered to be statistically significant. All analyses were performed using SPSS version 19.0 and GraphPad Prism version 8.2.1. 


\section{Results}

\subsection{Patient population}

Twenty patients were included in the study from March 2019 to April 2021. Demographic, clinical, and echocardiographic characteristics of these patients are shown in Table 1. Mean age was $67.0 \pm 11.2$ years. Patients included in the study had a tendency to have a long history of AF, and the mean CHA2DS2-VASc score was $1.9 \pm 1.2$. In addition to enlargement of the left atria, which was often seen in patients with $\mathrm{AF}$, patients in the study also appeared to have larger right atriums $(51.7 \pm 8.3 \times 42.9 \pm 3.7 \mathrm{~mm})$.

\subsection{Bi-atrial mapping}

Average sampling points of bi-atrial high precision mapping were $1880 \pm 445$, and the mean mapping time was $16.9 \pm 4.0 \mathrm{~min}$. Based on the specificity of RAA and its surrounding anatomical structures, site $A$, site $B$, and site $C$ were used to represent the septal side of the RAA base, the free wall side of the RAA base, and the inside of the RAA, respectively. AF cycle lengths in different segments of the left atrium and right atrium and their representative potential maps are shown in Table 2, Fig. 1, Fig. 2, and Fig. 3. The area with the shortest local average AFCL was RAA during bi-atrial mapping $(134.0 \pm 10.9 \mathrm{~ms})$. The roof as the area with the fastest potential frequency in the left atrium, its local AFCL $(145.5 \pm 14.9 \mathrm{~ms})$ is slightly longer than that of RAA. For the left atrium, potential frequencies of the LAA $(153.7 \pm 17.1 \mathrm{~ms})$ and bottom $(154.8 \pm 11.8 \mathrm{~ms})$ were also relatively fast, although slightly slower than that of the

Table 1 Characteristics of patients $(N=20)$

\begin{tabular}{ll}
\hline Male & $13(65.0)$ \\
\hline Age, years & $67.0 \pm 11.2$ \\
Hypertension & $9(45.0)$ \\
Diabetes mellitus & $2(10.0)$ \\
Coronary heart disease & $2(10.0)$ \\
$\mathrm{CHA}_{2} \mathrm{DS}_{2}$-VASc score & $1.9 \pm 1.2$ \\
Atrial fibrillation & \\
$\quad$ History of atrial fibrillation, months & $31(12,90)$ \\
$\quad$ Paroxysmal atrial fibrillation & $3(15.0)$ \\
$\quad$ Persistent atrial fibrillation & $9(45.0)$ \\
$\quad$ Long-standing persistent atrial fibrillation & $8(40.0)$ \\
$\quad$ Left atrial diameter, mm & $43.1 \pm 4.9$ \\
$\quad$ Right atrial diameter, mm & $51.7 \pm 8.3 \times 42.9 \pm 3.7$ \\
$\quad$ Left ventricular ejection fraction, $\%$ & $62.9 \pm 6.0$ \\
$\quad$ Complications & $0(0)$ \\
\hline
\end{tabular}

Values are $N(\%)$, mean $\pm \mathrm{SD}$, or median (25th to 75 th quartiles) roof. The above results are presented in Fig. 4. In the present study, the fastest frequency potentials were located within the RAA in $65 \%$ of the enrolled patients, and the proportions of the fastest frequency potentials at the septal side and free wall side of the RAA base were $25 \%$ and $10 \%$, respectively (Supplemental Fig. 1).

\subsection{Ablation outcomes}

Complete CPVI was achieved in all included patients. When using a PentaRay catheter to map the RAA, AF was terminated to sinus rhythm in three patients due to the mechanical stimulation of the catheter. These three patients had paroxysmal AF. Another 17 patients underwent RAA ablation, all at the RAA base, and the mean ablation time for RAA was $14.6 \pm 8.1 \mathrm{~min}$. According to the results of the mapping, the fastest frequency potentials were located at the free wall side of the RAA base in two patients, and AF was terminated after ablation in local patches, with one patient converting to sinus rhythm and the other to AFL. The mean ablation area of the RAA base was $318.0 \pm 25.5 \mathrm{~mm}^{2}$, and the mean ablation time for RAA was $19.0 \pm 1.4 \mathrm{~min}$. AF was terminated in five patients after sheet ablation only at the septal side of the RAA base, with two patients converting to sinus rhythm and the others to AFL. The mean ablation area of the RAA base was $86.4 \pm 19.7 \mathrm{~mm}^{2}$, and the mean ablation time for RAA was $6.0 \pm 1.6 \mathrm{~min}$. Mapping results of the other 10 patients showed that the high-frequency potentials were located in the RAA, so linear ablation was applied to the base of the RAA (both free wall side and septal side). The mean ablation area of the RAA base was $301.2 \pm 136.0 \mathrm{~mm}^{2}$, and the mean ablation time for RAA was $18.1 \pm 7.6 \mathrm{~min}$. AF was terminated after ablation with one patient converting to sinus rhythm and the others to AFL (Video). None of the patients had complications such as pericardial tamponade or thromboembolism. Representative ablation maps are shown in Supplemental Fig. 2.

\subsection{The electro-anatomical relationship between RAA and atrium}

According to the observation of the structure within RAA by ICE, we found that there were thick SBs along the long axis of RAA, which were formed by the convergence of multiple muscle bundles from TC. They went distally to the RAA and gave off branches reflexed to connect with the vestibule between the tricuspid annulus and the RAA, forming a circular muscular structure (Fig. 5A, B). In three patients with paroxysmal AF, we paced at the yellow spot which was located at the distal of SB after they transitioned to sinus rhythm, and the LAT mapping showed that the activation was transmitted from SB to $\mathrm{TC}$, to right atrial septum, and finally to left atrium by 


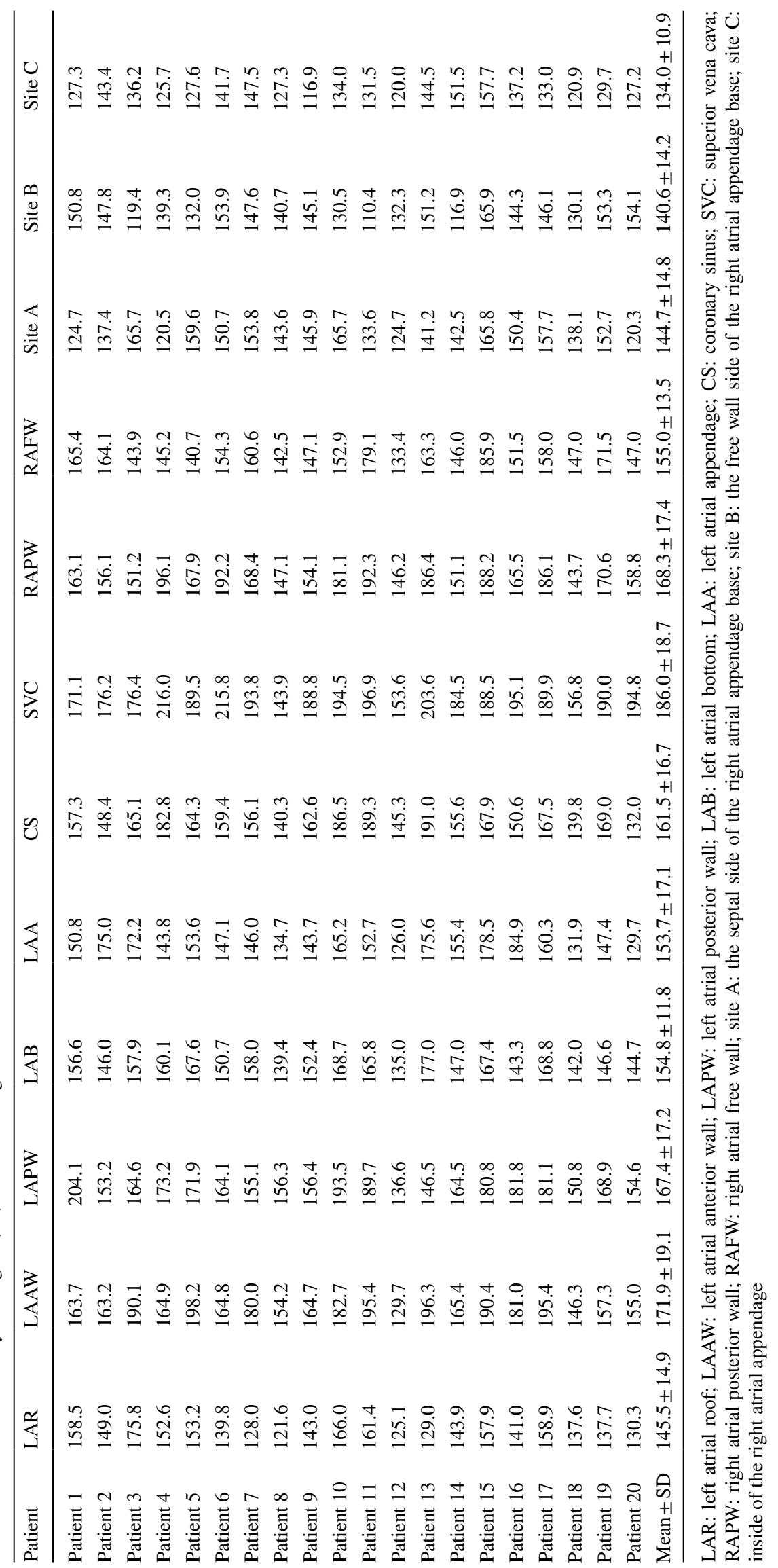




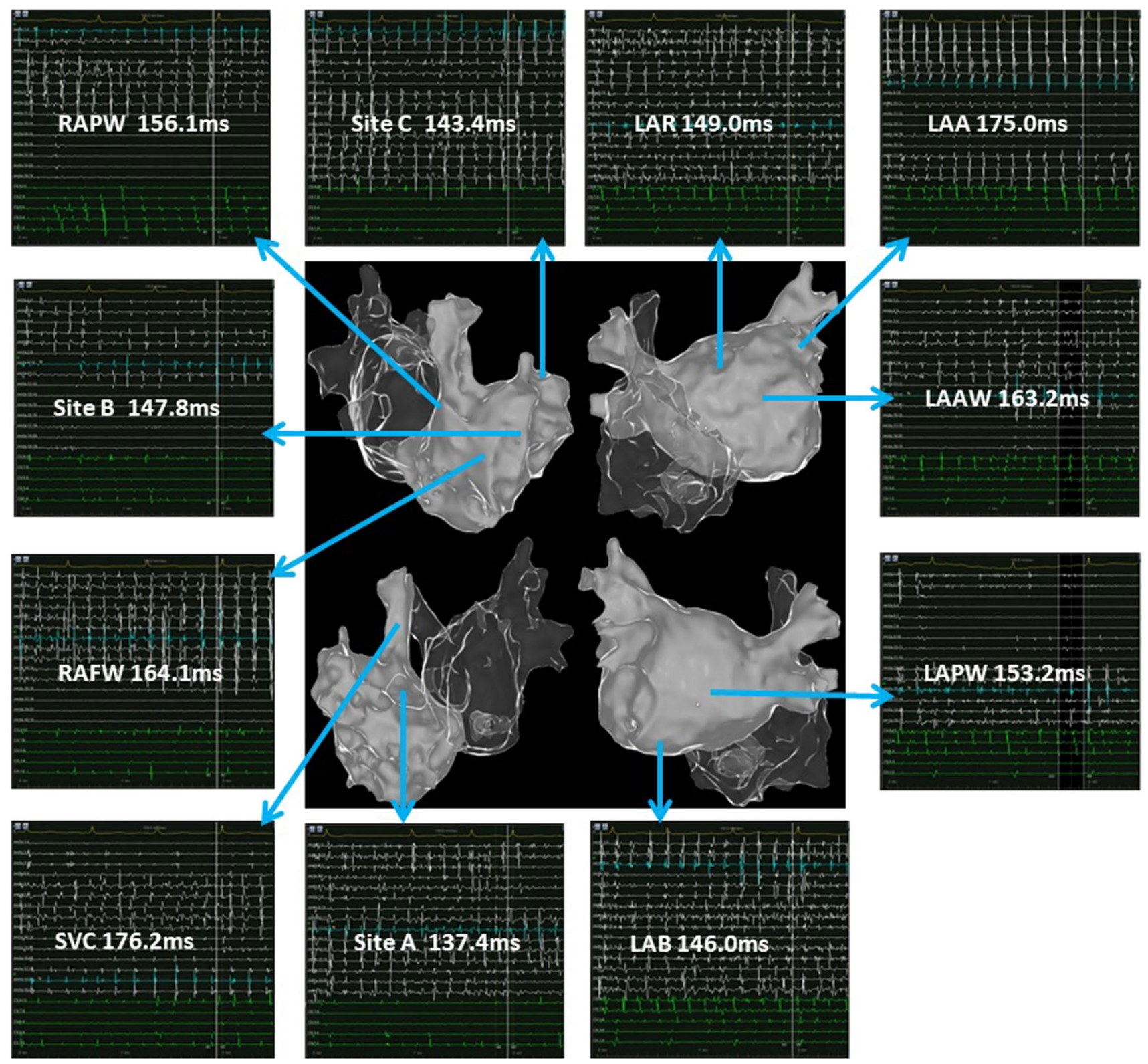

Fig. 1 Potential maps of different regions of the left atrium and right atrium in patient 2 . The potential frequency at the septal side of the right atrial appendage base was the fastest. For the left atrium, the potential frequency of the roof and bottom was relatively fast. In the potential maps, the white potential lines represent the bipolar potentials of the PentaRay electrodes and the green potential lines represent the bipolar potentials of the coronary sinus electrodes. Val-

Bachmann's bundle (BB) (Fig. 5C-E). The mean LAT from SB pacing to the earliest point of left atrium activation was $55.0 \pm 5.0 \mathrm{~ms}$, which was significantly shorter than the mean bi-atrial activation time $(176.7 \pm 10.3 \mathrm{~ms}$, $P<0.0001)$. This excitation feature suggested a dominant conduction pathway among the RAA, TC, and BB, which was consistent with the trend of bipolar potential frequency measured in AF. ues represent the atrial fibrillation cycle lengths in the corresponding regions. LAR: left atrial roof; LAAW: left atrial anterior wall; LAPW: left atrial posterior wall; LAB: left atrial bottom; LAA: left atrial appendage; SVC: superior vena cava; RAPW: right atrial posterior wall; RAFW: right atrial free wall; site A: the septal side of the right atrial appendage base; site B: the free wall side of the right atrial appendage base; site $\mathrm{C}$ : inside of the right atrial appendage

\subsection{Follow-up}

Because of the different dates of ablation, not all patients completed the four post-ablation follow-ups. All patients completed 1-, 3-, and 6-month follow-up after ablation, and the number of patients who completed 9and 12-month follow-up after ablation was 16 and 13 . No patients have had recurrent AF so far, which was a 


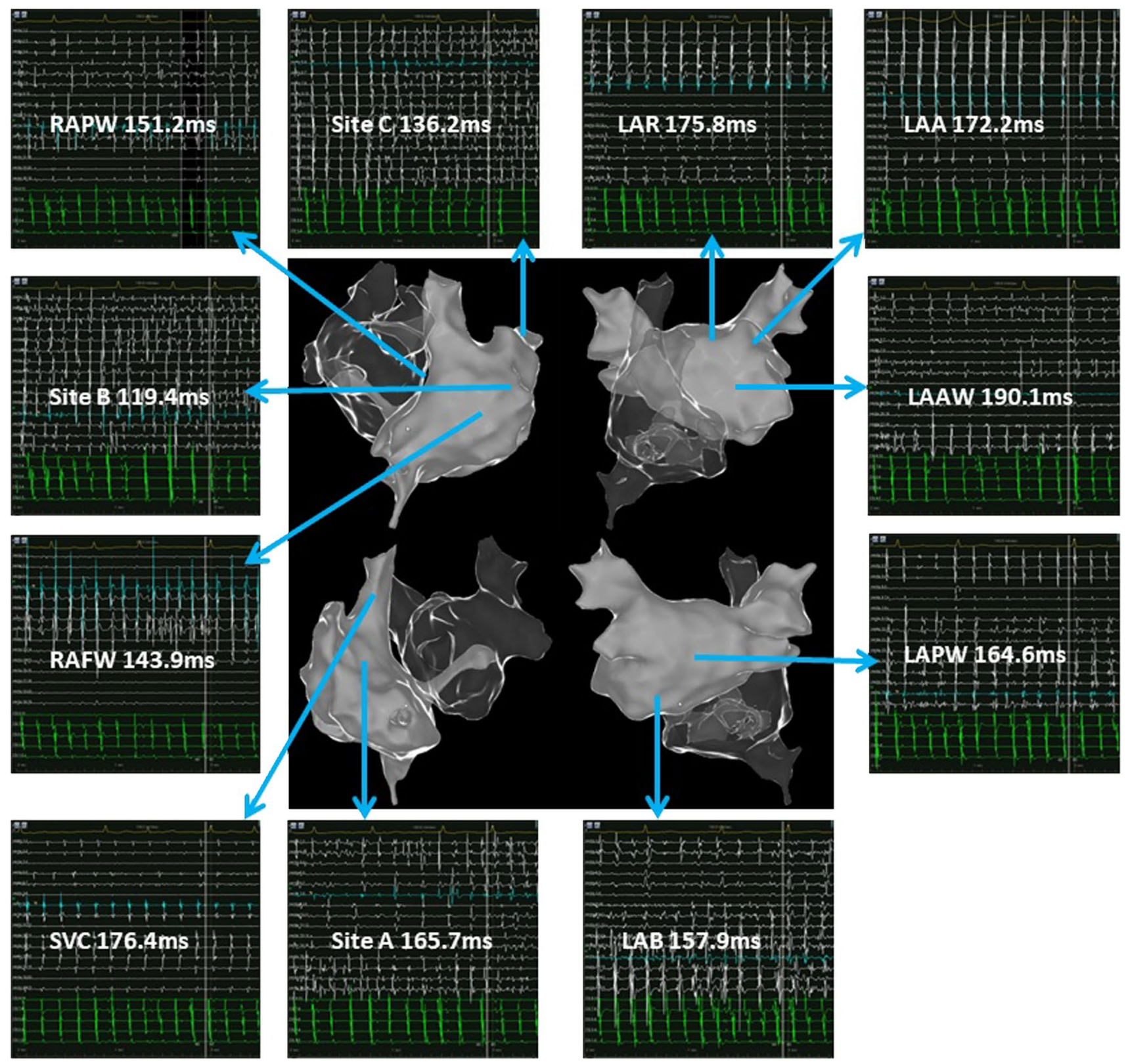

Fig. 2 Potential maps of different regions of the left atrium and right atrium in patient 3 . The potential frequency at the free wall side of the right atrial appendage base was the fastest. For the left atrium, the potential frequency of the bottom was the fastest. In the potential maps, the white potential lines represent the bipolar potentials of the PentaRay electrodes and the green potential lines represent the bipolar potentials of the coronary sinus electrodes. Values represent the atrial fibrillation cycle lengths in the corresponding regions. LAR: left atrial roof; LAAW: left atrial anterior wall; LAPW: left atrial posterior wall; LAB: left atrial bottom; LAA: left atrial appendage; SVC: superior vena cava; RAPW: right atrial posterior wall; RAFW: right atrial free wall; site A: the septal side of the right atrial appendage base; site B: the free wall side of the right atrial appendage base; site $\mathrm{C}$ : inside of the right atrial appendage satisfactory result. In terms of maintaining sinus rhythm, one patient had AFL and 95.0\% (19/20) of including patients maintained sinus rhythm at 1 month and 3 months after ablation. Six months after ablation, one patient who maintained sinus rhythm during the previous two follow-ups had AFL, and 90.0\% (18/20) of patients maintained sinus rhythm. Sinus rhythm was maintained in $87.5 \%$ and $84.6 \%$ of patients at 9 and 12 months after ablation, respectively. The above results are shown in Supplemental Fig. 3. 


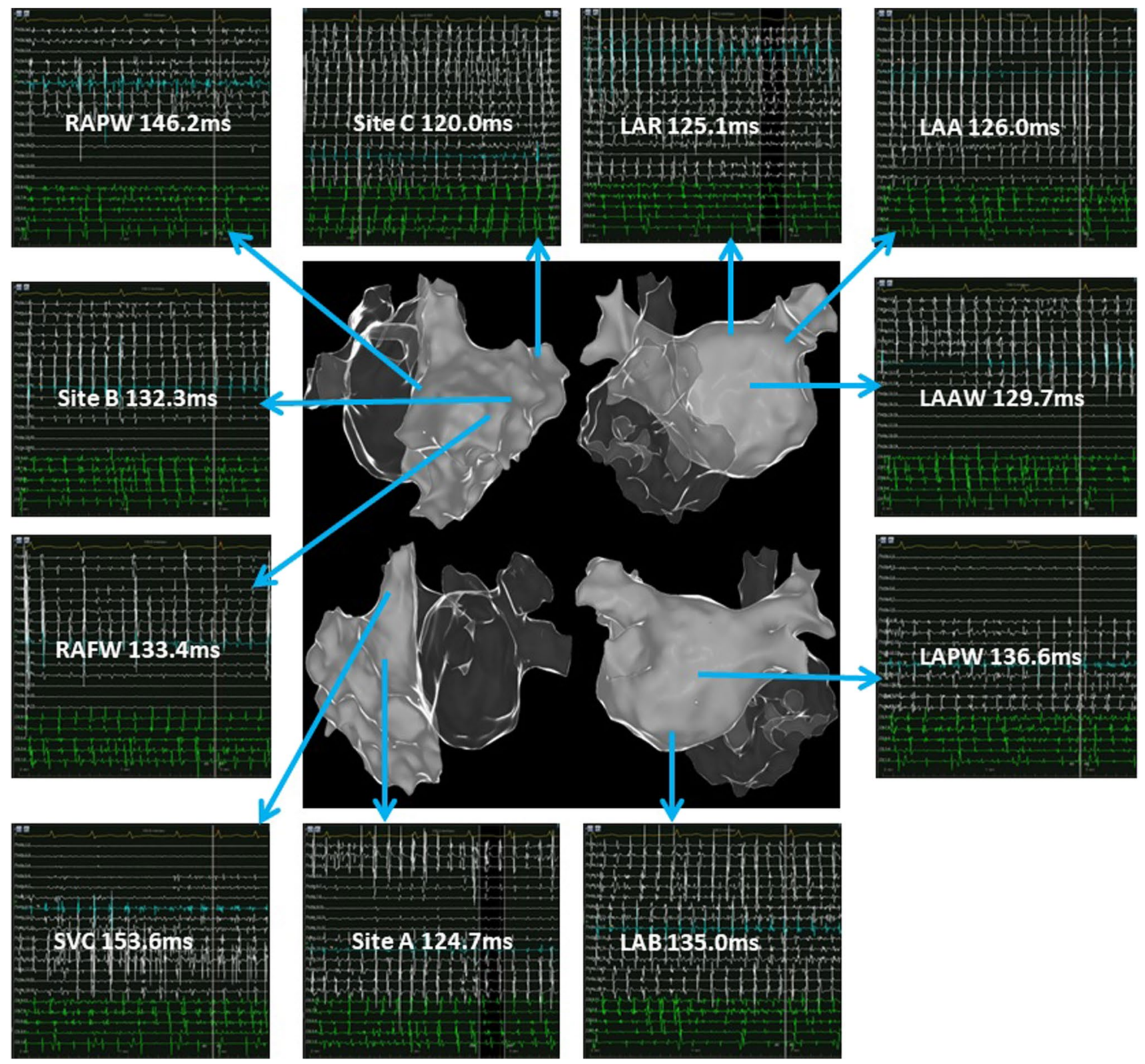

Fig. 3 Potential maps of different regions of the left atrium and right atrium in patient 12 . The potential frequency inside the right atrial appendage was the fastest. For the left atrium, there were obvious high-frequency potentials at the roof, bottom, and left atrial appendage. In the potential maps, the white potential lines represent the bipolar potentials of the PentaRay electrodes and the green potential lines represent the bipolar potentials of the coronary sinus electrodes.

\section{Discussion}

The RAA is an important substrate for the maintenance of $\mathrm{AF}$, and its role is often neglected. This study found the relationship between the atrial potential characteristics and anatomical characteristics of the AF driven by RAA. The SBTC-BB dominant conduction pathway which could transmit high-frequency potentials from the RAA to the left atrium

Values represent the atrial fibrillation cycle lengths in the corresponding regions. LAR: left atrial roof; LAAW: left atrial anterior wall; LAPW: left atrial posterior wall; LAB: left atrial bottom; LAA: left atrial appendage; SVC: superior vena cava; RAPW: right atrial posterior wall; RAFW: right atrial free wall; site A: the septal side of the right atrial appendage base; site B: the free wall side of the right atrial appendage base; site $\mathrm{C}$ : inside of the right atrial appendage

was the structural basis of this characteristic. The termination of AF could be achieved through ablation and improvement of this substrate.

The RAA forms the anterior wall of the atrium and is composed of ridges formed by pectinate muscles, which arise from the prominent crista terminalis [6]. A previous study which was carried out on the basis of echocardiography imaging reported that the area of RAA was bigger 


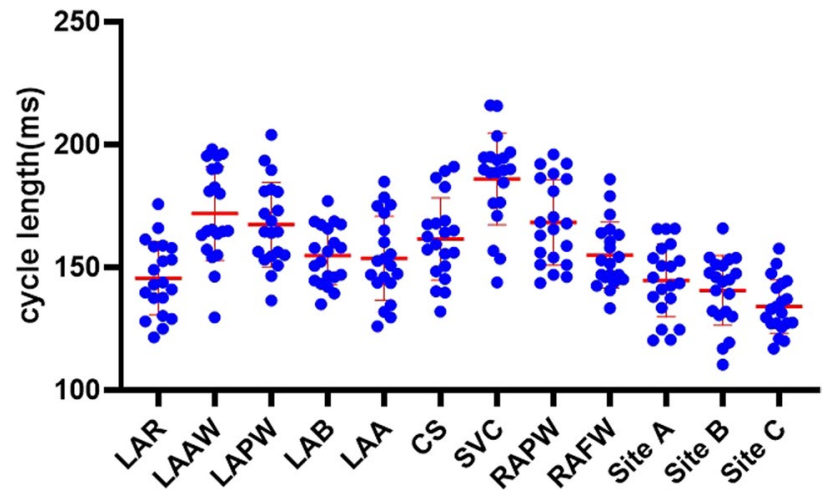

Fig. 4 Scatter diagram of the atrial fibrillation cycle lengths in different regions of the left and right atrium. LAR: left atrial roof; LAAW: left atrial anterior wall; LAPW: left atrial posterior wall; LAB: left atrial bottom; LAA: left atrial appendage; CS: coronary sinus; SVC: superior vena cava; RAPW: right atrial posterior wall; RAFW: right atrial free wall; site A: the septal side of the right atrial appendage base; site B: the free wall side of the right atrial appendage base; site $\mathrm{C}$ : inside of the right atrial appendage

in patients with $\mathrm{AF}$ than in patients with sinus rhythm [7]. Besides, other studies indicated that RAA fibrosis and varied shape had an impact on the occurrence and maintenance of AF [8-11]. In our cases, the right atriums were enlarged to varying degrees, and the special anatomical structure in the RAA was also the key factor for the formation of AF driver. A study on optical mapping of human RAA showed that architectural discontinuity between pectinate muscles and small intramural bundles could lead to longitudinal and intramural conduction blocks and increased fibrosis could worsen the architectural discontinuity, which ultimately could create a structural substrate for AF driver [12]. This suggested that the RAA had anatomic substrates for $\mathrm{AF}$ formation.

The involvement of the right atrium in the maintenance of $\mathrm{AF}$ has been found in previous clinical studies [2, 13], and similar findings had been reported in our previous study [14]. However, the proportion of right atrial driver was low. This may be related to the non-routine bi-atrial mapping and the neglect of the role of RAA. In our study, we found that in the AF driven by RAA, the left atrial local potential also presented a certain degree of high-frequency potentials, especially at the BB insertion point of the roof and within LAA. This phenomenon may misjudge the results of the mapping and result in left atrial excessive ablation. In 13 (65\%) cases in our study, the true driver of AF was found to be located in RAA after the corresponding region of the left atrium was ablated first. The mechanism of this potential characteristic was due to the existence of SB-TC-BB dominant conduction pathway. It has been shown that the conduction between the atria occurs mainly in BB [15]. The superior arm of the rightward extension of $\mathrm{BB}$ arises in the vicinity of the $\mathrm{SB}$, and the inferior arm arises in the subepicardium of the right atrial vestibule [16]. The left side of BB is inserted into the anterior top of the left atrium and extends to the LAA [17]. In our study, through pacing RAA, it was found that the conduction time of SB to the position of the junction between $\mathrm{BB}$ and left atrium was significantly shorter than the activation time of other parts of the atrium, indicating that driver of RAA could rapidly spread to the left atrium through this pathway. This is also why the potential frequencies in other parts of the right atrium were slower than that of the left atrial roof. Notably, interatrial bundles are not limited to the BB and are present in all areas of the interatrial septum. Besides, inferiorly located bundles can sometimes be more prominent than the BB [18]. This explains why the frequency of the left atrial bottom potential was very fast in some patients.

Our strategy for ablation of RAA is as follows: (1) For AF driven by the base of RAA, ablation was performed in sheet form at the corresponding septal or free wall side of the RAA base. (2) For cases where the driver was located in the RAA, the dominant SB-BB pathway was blocked by linear ablation at the septal and free wall side of the RAA base. Immediate termination of AF was achieved by this ablation strategy, although the driver in the RAA was not directly interfered with and this linear ablation was difficult to achieve complete blockade of conduction. We speculated that it may be due to the substrate improvement effect. In a previous study [13], ablation in the RAA was guided by the Lasso catheter, which was inserted into the body of the RAA, with ablation proximal to the catheter, and this method was reported to be able to terminate AF. The ablation method recently reported by Ghannam et al. was circumferential ablation around the RAA [2]. Because of the fact that RAA has pectinate, smooth-walled vestibule and low blood flow [19], catheter ablation within RAA is complicated. In addition, the shallow nature of the atrial appendage increases the likelihood that current catheter manipulation within RAA may easily lead to cardiac perforation. Thus, compared with the above two methods, our ablation method is safer and reduces ablation damage while terminating AF. Certainly, we look forward to better intervention techniques for the atrial appendage in the future.

\subsection{Limitations}

First, the number of cases contained in the present study was relatively small, and we did not set a propensity score matched group, which in our opinion would not be significant for such a small sample. Since the main purpose of this study was to summarize the characteristics and ablation methods of the AF driven by RAA, a control group was not very necessary. Second, the relationship between the morphology of RAA and AF has not been carefully studied due 
Fig. 5 A, B Structures in the right atrial appendage under intracardiac echocardiography. The blue arrows indicate the sagittal bundle. : terminal crest; and : sagittal bundle. C, D Results of local activation time mapping when paced at the yellow spot in the right atrial appendage. For this patient, the conduction time from SB pacing to the earliest point of left atrium activation was $55 \mathrm{~ms}$, and the bi-atrial activation time was $174 \mathrm{~ms}$. The corresponding data of the other two patients who underwent local activation time mapping were $60 \mathrm{~ms}, 188 \mathrm{~ms}$ and $50 \mathrm{~ms}$, $168 \mathrm{~ms}$, respectively. E The activation in the right atrial appendage (marked by the red star) was transmitted from sagittal bundle to terminal crest, to right atrial septum and finally to left atrium by Bachmann's bundle. SB: sagittal bundle; TC: terminal crest; BB: Bachmann's bundle; SVC: superior vena cava; IVC: inferior vena cava; LAA: left atrial appendage; CS: coronary sinus

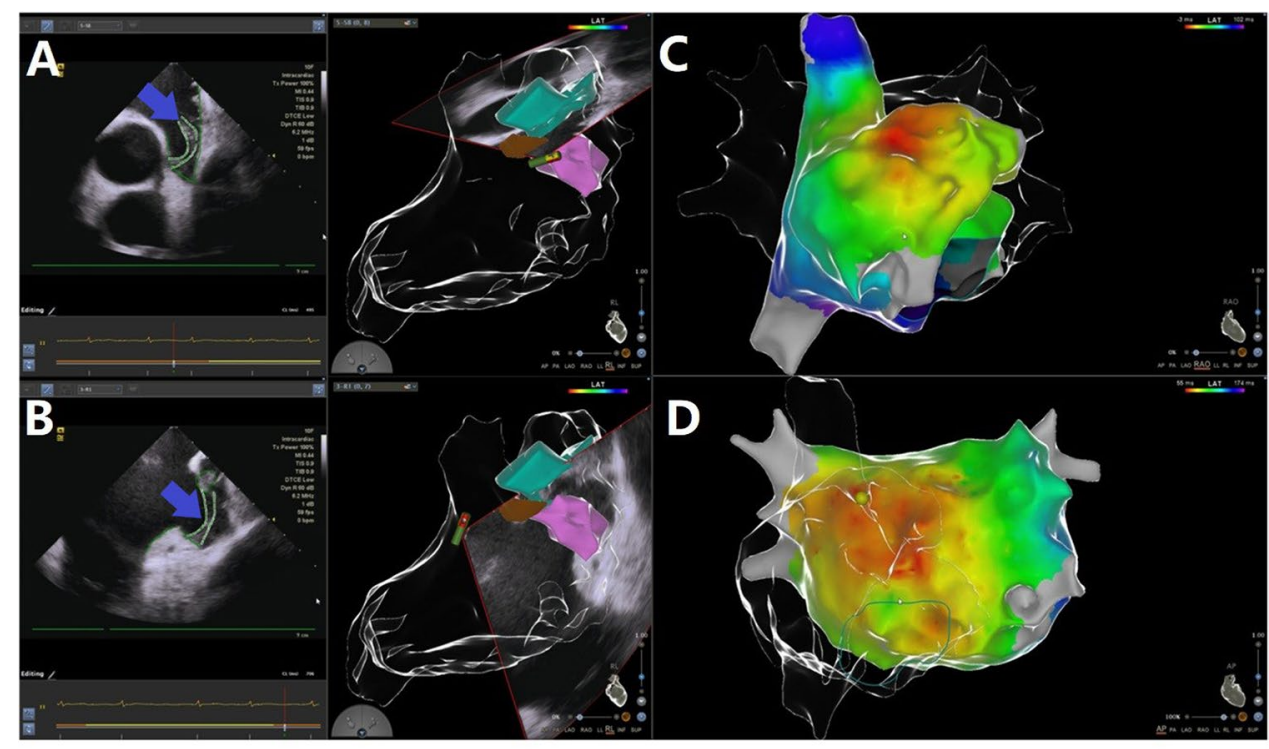

$\mathbf{E}$

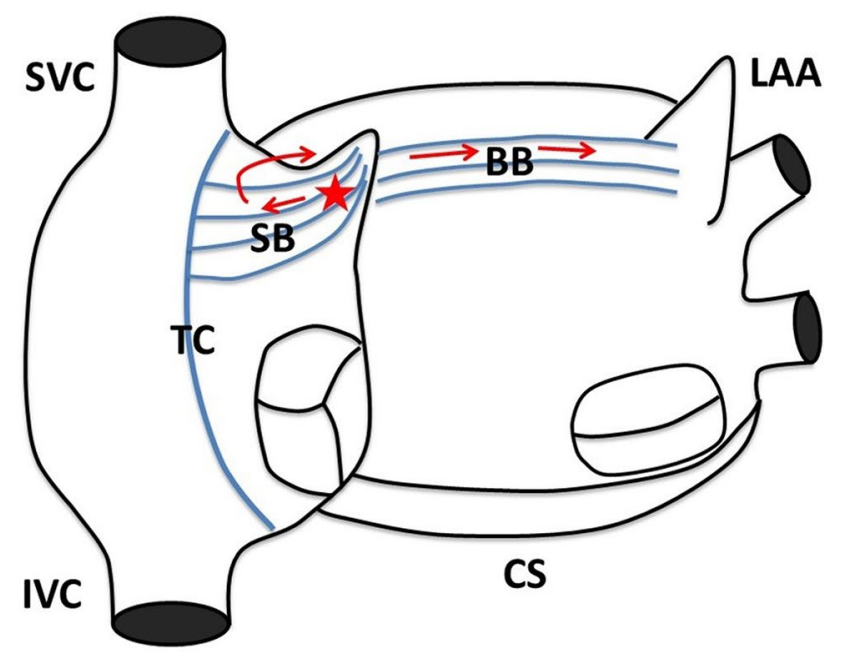

to the absence of some relevant materials. Last but not least, further studies are needed to confirm the efficacy of this ablation approach and to determine the detailed mechanism and role of RAA ablation in AF.

\section{Conclusions}

The left atrial potential characteristics of the AF driven by RAA, which was related to the SB-TC-BB dominant conduction pathway between the RAA and left atrium, may easily lead to the misjudgment of drivers. For this type of AF, ablation at the RAA base could lead to the termination of AF in a safe and long-term manner.

Supplementary Information The online version contains supplementary material available at https://doi.org/10.1007/s10840-021-01106-8.
Acknowledgements This research was supported by Grants from the National Natural Science Foundation of China (NO. 81770324) and National Key Research and Development Project (Grant Number 2018YFC1312503).

\section{Declarations}

Ethics approval This study protocol was approved by the Institutional Ethics Committee at the Shanghai Chest Hospital.

Informed consent Not applicable.

Conflict of interest The authors declare no competing interests.

Open Access This article is licensed under a Creative Commons Attribution 4.0 International License, which permits use, sharing, adaptation, distribution and reproduction in any medium or format, as long as you give appropriate credit to the original author(s) and the source, provide a link to the Creative Commons licence, and indicate if changes were made. The images or other third party material in this article are 
included in the article's Creative Commons licence, unless indicated otherwise in a credit line to the material. If material is not included in the article's Creative Commons licence and your intended use is not permitted by statutory regulation or exceeds the permitted use, you will need to obtain permission directly from the copyright holder. To view a copy of this licence, visit http://creativecommons.org/licenses/by/4.0/.

\section{References}

1. Santangeli P, Zado ES, Hutchinson MD, Riley MP, Lin D, Frankel DS, et al. Prevalence and distribution of focal triggers in persistent and long-standing persistent atrial fibrillation. Heart Rhythm. 2016;13(2):374-82. https://doi.org/10.1016/j.hrthm.2015.10.023.

2. Ghannam M, Jame S, Jongnarangsin K, Cheng YW, Gunda S, Fadahunsi $\mathrm{O}$, et al. Catheter ablation of the left and right atrial appendages without isolation in persistent atrial fibrillation. Heart Rhythm. 2021;18(5):694-701. https://doi.org/10.1016/j.hrthm. 2021.01.006

3. Schuessler RB, Kawamoto T, Hand DE, Mitsuno M, Bromberg BI, Cox JL, et al. Simultaneous epicardial and endocardial activation sequence mapping in the isolated canine right atrium. Circulation. 1993;88(1):250-63. https://doi.org/10.1161/01.cir.88.1.250.

4. Eckstein J, Maesen B, Linz D, Zeemering S, van Hunnik A, Verheule $\mathrm{S}$, et al. Time course and mechanisms of endo-epicardial electrical dissociation during atrial fibrillation in the goat. Cardiovasc Res. 2011;89(4):816-24. https://doi.org/10.1093/cvr/cvq336.

5. Matsuo S, Lellouche N, Wright M, Bevilacqua M, Knecht S, Nault I, et al. Clinical predictors of termination and clinical outcome of catheter ablation for persistent atrial fibrillation. J Am Coll Cardiol. 2009;54(9):788-95. https://doi.org/10.1016/j.jacc.2009. 01.081 .

6. Anderson RH, Cook AC. The structure and components of the atrial chambers. Europace. 2007;9(Suppl 6):vi3-9. https://doi.org/ 10.1093/europace/eum200.

7. de Divitiis M, Omran H, Rabahieh R, Rang B, Illien S, Schimpf $\mathrm{R}$, et al. Right atrial appendage thrombosis in atrial fibrillation: its frequency and its clinical predictors. Am J Cardiol. 1999;84(9):1023-8. https://doi.org/10.1016/s0002-9149(99) 00492-0.

8. van Brakel TJ, van der Krieken T, Westra SW, van der Laak JA, Smeets JL, van Swieten HA. Fibrosis and electrophysiological characteristics of the atrial appendage in patients with atrial fibrillation and structural heart disease. J Interv Card Electrophysiol. 2013;38(2):85-93. https://doi.org/10.1007/s10840-013-9820-8.

9. Kostin S, Klein G, Szalay Z, Hein S, Bauer EP, Schaper J. Structural correlate of atrial fibrillation in human patients. Cardiovasc
Res. 2002;54(2):361-79. https://doi.org/10.1016/s0008-6363(02) 00273-0.

10. Kaminski R, Grzybiak M, Nowicka E, Kosinski A, Lewicka E, Dabrowska-Kugacka A, et al. Macroscopic morphology of right atrial appendage in humans. Kardiol Pol. 2015;73(3):183-7. https://doi.org/10.5603/KP.a2014.0170.

11. Rissi R, Marques MJ, Neto HS. Checking the shape and lobation of the right atrial appendage in view of their clinical relevance. Anat Sci Int. 2019;94(4):324-9. https://doi.org/10.1007/ s12565-019-00489-z.

12. Hansen BJ, Zhao J, Li N, Zolotarev A, Zakharkin S, Wang Y, et al. Human atrial fibrillation drivers resolved with integrated functional and structural imaging to benefit clinical mapping. JACC Clin Electrophysiol. 2018;4(12):1501-15. https://doi.org/ 10.1016/j.jacep.2018.08.024.

13. Hocini M, Nault I, Wright M, Veenhuyzen G, Narayan SM, Jais P, et al. Disparate evolution of right and left atrial rate during ablation of long-lasting persistent atrial fibrillation. J Am Coll Cardiol. 2010;55(10):1007-16. https://doi.org/10.1016/j.jacc.2009.09.060.

14. Qin M, Jiang WF, Wu SH, Xu K, Liu X. Electrogram dispersionguided driver ablation adjunctive to high-quality pulmonary vein isolation in atrial fibrillation of varying durations. J Cardiovasc Electrophysiol. 2020;31(1):48-60. https://doi.org/10.1111/jce. 14268.

15. Enriquez A, Conde D, Hopman W, Mondragon I, Chiale PA, de Luna $A B$, et al. Advanced interatrial block is associated with recurrence of atrial fibrillation post pharmacological cardioversion. Cardiovasc Ther. 2014;32(2):52-6. https://doi.org/10.1111/ 1755-5922.12063.

16. van Campenhout MJ, Yaksh A, Kik C, de Jaegere PP, Ho SY, Allessie MA, et al. Bachmann's bundle: a key player in the development of atrial fibrillation? Circ Arrhythm Electrophysiol. 2013;6(5):1041-6. https://doi.org/10.1161/CIRCEP.113.000758.

17. Khaja A, Flaker G. Bachmann's bundle: does it play a role in atrial fibrillation? Pacing Clin Electrophysiol. 2005;28(8):855-63. https://doi.org/10.1111/j.1540-8159.2005.00168.x.

18. Platonov PG, Mitrofanova L, Ivanov V, Ho SY. Substrates for intra-atrial and interatrial conduction in the atrial septum: anatomical study on 84 human hearts. Heart Rhythm. 2008;5(8):1189-95. https://doi.org/10.1016/j.hrthm.2008.04.025.

19. Anderson RH, Razavi R, Taylor AM. Cardiac anatomy revisited. J Anat. 2004;205(3):159-77. https://doi.org/10.1111/j.0021-8782. 2004.00330.x.

Publisher's note Springer Nature remains neutral with regard to jurisdictional claims in published maps and institutional affiliations. 